\title{
Nanoporous gallium nitride square microtubes
}

\author{
Yucheng Lan · Hui Wang · Feng Lin • \\ Yalin Lu • Yang Li $\cdot$ Yuan Liu · Jiming Bao • \\ Zhifeng Ren $\cdot$ Martin A. Crimp
}

Received: 2 April 2013/Accepted: 9 July 2013/Published online: 23 July 2013

(C) Springer Science+Business Media New York 2013

\begin{abstract}
Porous gallium nitride microtubes were selffabricated from gallium nitride submicron irregular structures. The microtubes were of square cross-section. Electron diffractions indicated that the microtubes were composed of zincblende gallium nitride. Electron energy-loss spectrum and photoluminescence spectrum of the microtubes were collected and compared with that of single crystals.
\end{abstract}

\section{Introduction}

Gallium nitride $(\mathrm{GaN})$ semiconductors have a direct bandgap of 3.2-3.4 eV and have found wide applications in blue and UV light emission devices and laser devices [1,2]. To date, GaN nanoparticles [3], nanorods [4, 5], nanowires [6-9], nanotubes [10-13], as well as single crystals [14-16]

Y. Lan $(\bowtie) \cdot$ H. Wang $\cdot$ Y. Liu $\cdot$ Z. Ren

Department of Physics and Texas Center for Superconductivity, University of Houston, Houston, TX 77204, USA

e-mail: ylan2@uh.edu

Z. Ren

e-mail: zren@uh.edu

F. Lin

State Key Laboratory for Mesoscopic Physics,

School of Physics, Peking University, Beijing 100871, China

Y. Lu

Laser Optics Research Center, US Air Force Academy,

Colorado Springs, CO 80840, USA

Y. Li · J. Bao

Department of Electrical and Computer Engineering, University of Houston, Houston, TX 77204, USA

M. A. Crimp

Department of Chemical Engineering and Material Science, Michigan State University, East Lansing, MI 48824, USA have been synthesized using a variety of techniques. The quantum confinement of these nanomaterials yields unique physical properties over bulk crystals, making the $\mathrm{GaN}$ nanomaterials wider applications, such as on photocatalytic water splitting $[17,18]$ besides light emission devices and laser devices. However, it is still challenging to assemble $\mathrm{GaN}$ nanomaterials into macroscaled bulks for industrial applications.

Up to now there are three achievements on the selfassembly of $\mathrm{GaN}$ nanoparticles. It was reported that $\mathrm{GaN}$ nanoparticles (diameter of $\sim 12 \mathrm{~nm}$ ) can be self-assembled into millimeter scale bulk nanocomposites [19] under supercritical high-pressure solution environments. GaN nanoparticles were closely compacted into bulks under the supercritical conditions. GaN nanoparticles (diameter of several nanometers) were also self-assembled into nanospheres [20] with diameters of 20-25 nm or nanotubes [20] with shell thicknesses of $2.5-4.5 \mathrm{~nm}$ by a gas interface reaction route. Recently it was reported that wurtzite $\mathrm{GaN}$ nanorods were successfully assembled into micron scale tubular structures with diameters of about $10 \mu \mathrm{m}$ [21] under supersaturated conditions. All these bulky assemblies inhibited quantum confinement effects of nanomaterials.

Here we report another kind of $\mathrm{GaN}$ self-assemblies: porous $\mathrm{GaN}$ square microtubes. Such porous microtubes were found to be consisted of GaN submicron irregular structures with a zincblende structure. The self-assembled GaN square microbes possess both advances of bulks (macroscale size) and nanomaterials (quantum confinement effect).

\section{Experimental}

The synthesis of the microtubes was carried out in a horizontal quartz tube furnace. Gallium metal (99.999\% 
purity) was purchased from Sigma-Aldrich Corporation. Quartz substrates were washed by ultrasonicating in acetone and then in de-ionized water, followed by thorough rinsing with methanol and drying in air. Gallium was placed on quartz substrates and positioned at the center of the horizontal tube in a hot-walled chemical vapor deposition (CVD) furnace. The CVD system was evacuated by a mechanical pump backed molecule pump, and purged several times with argon gas before introducing ammonia gas. As-received analytical grade (purity of 99.9\%) commercial argon and ammonia gas were used without further purification. The entire system was heated from room temperature to $800^{\circ} \mathrm{C}$ in about $20 \mathrm{~min}$ and maintained at the reaction temperature for $2 \mathrm{~min}$. Afterwards, the flow of ammonia was stopped and the entire system was cooled to room temperature.

Scanning electron microscopy (SEM) images were taken on a CamScan FE 44 SEM at $25 \mathrm{kV}$. Transmission electron microscopy (TEM) experiments were carried out on a JEOL 2200FS TEM equipped with an in-column energy filter and electron energy-loss spectroscopic (EELS) spectra were collected in scanning transmission electron microscopy mode. The room temperature photoluminescence (PL) of GaN microtubes was performed using a 337-nm UV laser and a single-grating spectrometer (Horiba HR320).

\section{Results and discussion}

After reactions yellow deposits were observed on the substrates. SEM observation indicated that the yellow deposits consisted of many microtubes. Figure 1a-c shows
SEM images of some microtubes grown on the substrates. The synthesized structures were hollow microtubes with cross sections about $3 \mu \mathrm{m}$ and lengths up to $100 \mu \mathrm{m}$. The ends of most of these microtubes were open, but some were closed (Fig. 1a). SEM images also indicated that all tube walls were nearly equal in width (Fig. 1a-d) and all face angles were at right angles (Fig. 1a-e). That is, the synthesized microtubes were square in cross-section.

From the open ends of the microtubes (Fig. 1a-c), the thicknesses of the microtube walls were found to be about $100 \mathrm{~nm}$. More detailed examination (Fig. 1d) indicated the walls consisted of single layer of randomly oriented submicron irregular structures. The diameters of the submicron irregular structures were about $100 \mathrm{~nm}$ and their lengths were about $1 \mu \mathrm{m}$ (Fig. 1d-f).

High magnification SEM images (Fig. 1d-f) indicated that, as a result of the random packing of the submicron irregular structures, the thin walls of the microtubes were porous. Some nanopores of these walls are noted by the arrows in Fig. 1d-f. Such porous structures should have large surface areas. Unfortunately, it is very difficult to remove the synthesized microtubes from the substrates because of brittleness and their surface area was not measured experimentally.

Figure 2a shows a typical TEM image of the square microtubes at low magnification. The thin wall of the microtube is porous. Selected area electron diffraction (SAED) patterns (Fig. 2b) collected from the microtube indicated that the microtube was polycrystalline. The electron diffraction pattern can be indexed to cubic GaN.

In order to confirm the cubic crystallographic structure of the thin wall further, SAED patterns were also taken at nanoscale. Figure 2c shows a typical TEM image of a
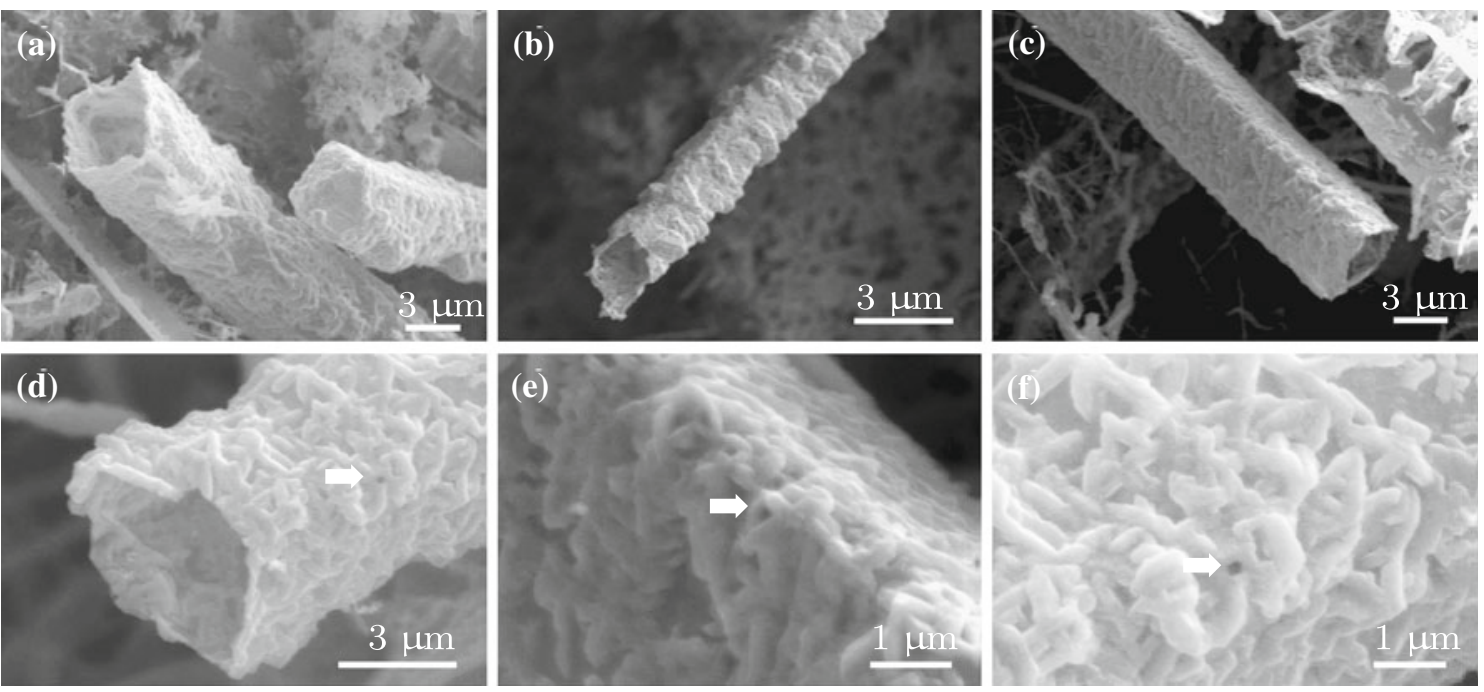

Fig. 1 SEM images of GaN square microtubes. a-c Microtubes grown at different regions. d End of one microtube. e Edge of a square microtube. $\mathbf{f}$ Enlarged surface of a microtube. White arrows mark the nanopores of the microtubes 
Fig. 2 a TEM image of a square microtube at low magnification. b SAED pattern of the circled region in a. The pattern is indexed as the cubic GaN phase. c TEM image of a $\mathrm{GaN}$ square microtube end at higher magnification. d SAED pattern of the circled region in c. The pattern is indexed as the cubic GaN phase with a zone axis of [111]
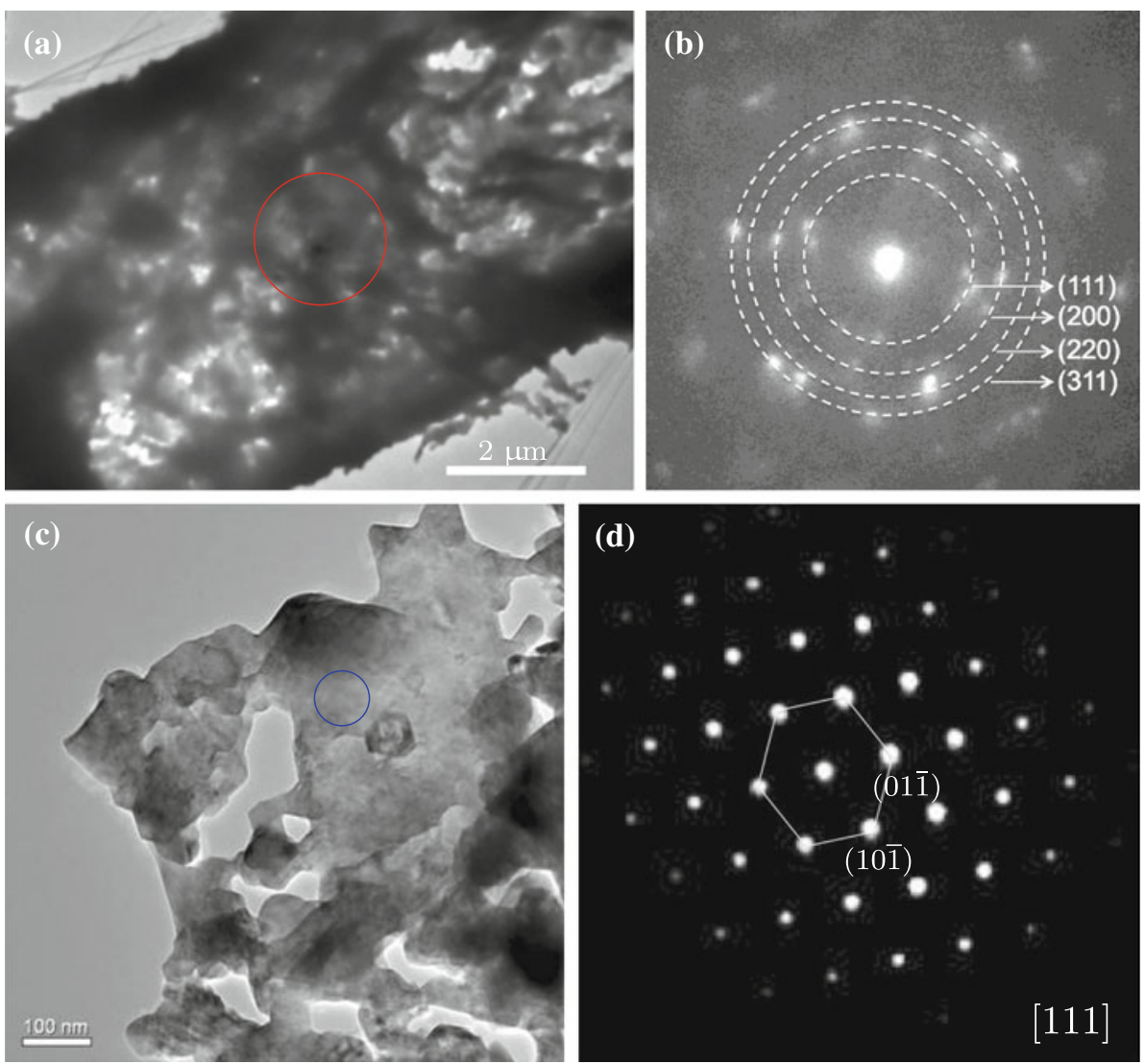

microtube square end at higher magnification. Nanopores were also observed from the TEM image. Figure $2 d$ is the SAED pattern of a nano-region. The nano-region is single crystalline. The pattern has a threefold symmetry and can be indexed as the cubic GaN (c-GaN) phase (space group of $\mathrm{F} \overline{4} 3 \mathrm{~m}$ with a lattice parameter of $4.503 \AA$ ) with a zone axis of [111] or the hexagonal GaN (h-GaN) phase (space group of $\mathrm{P} 6_{3} \mathrm{mc}$ with lattice parameters of $a=3.18907 \AA$ and $c=5.1855 \AA$ ) with a zone axis of [001]. The interplane distance $d$ corresponding to the diffracted spots was carefully measured from Fig. $2 \mathrm{~d}$. The measured $d$ values of the diffracted spots are 3.3 and $1.6 \AA$, corresponding to that of $(110)\left(d_{(110)}=3.18 \AA\right)$ and $(220)\left(d_{(220)}=1.59 \AA\right)$, respectively, of c-GaN with the zinc blende structure, while far from that of (100) $\left(d_{(100)}=2.76 \AA\right)$ and (200) $\left(d_{(200)}=1.38 \AA\right)$ of the h-GaN with the wurtzite crystal structure. Therefore, the SAED pattern confirmed that the wall nanoregion is of c-GaN with a zone axis of [111], not h-GaN with a zone axis of [001]. Therefore the entire microtubes are polycrystalline while the consisting irregular nanostructures are single crystalline c-GaN.

Figure 3a shows an EELS spectrum revealing the lowloss region and the zero-loss peak (ZLP) of a GaN microtube. The energy resolution of the EELS spectrum is $1.75 \mathrm{eV}$, according to the full-width at half-maximum of the zero loss peak. A plasmon excitation is determined at $\sim 25 \mathrm{eV}$. This plasmon peak position is very close to that of $\mathrm{GaN}$ bulks [22, 23]. It has been reported that the plasmon peak position shifts linearly with strain [24] caused by confinement size and defects in GaN nanomaterials ( $0.168 \mathrm{eV}$ per $1 \%$ volume change). However such subtle plasmon peak shifts are not observed here because of the limited EELS energy resolution.

Figure $3 \mathrm{~b}$ shows the electron-energy loss near-edge fine structures (ELNES) of the nitrogen $\mathrm{K}$ edge. Three main peaks are observed, at 403, 405, and $407 \mathrm{eV}$. Compared with the standard data of $\mathrm{GaN}$ crystals (400, 403.5, $405.5 \mathrm{eV}[25,26])$, the loss peaks shift slightly. Figure $3 \mathrm{c}$ shows the ELNES of the gallium $\mathrm{L}_{2,3}$ edge, which arises from the excitation of electrons from the $2 p_{3 / 2}$ and $2 p_{1 / 2}$ states to $(4 s, 4 d)$ states in the conduction band. The Ga- $\mathrm{L}_{2,3}$ edges reflect the $s$ and $d$ partial unoccupied density of states. The energy of the $\mathrm{L}_{3}$ edge is very close to the reported value of single crystalline $\mathrm{GaN}(1125 \mathrm{eV})$ [22]. However, the observed peak position of the $\mathrm{Ga}-\mathrm{L}_{2}$ $(1175 \mathrm{eV})$ was very different from the reported values for bulk GaN (1126 eV [26] or $1155 \mathrm{eV}$ [22]). The difference in the Ga- $\mathrm{L}_{2}$ edge should come from the confinement effect of the $\mathrm{GaN}$ submicron irregular structures, which change the $s$ unoccupied density of states of the microtubes.

Figure 4 show a PL spectrum of the square microtubes. A strong and wide yellow band was observed at 

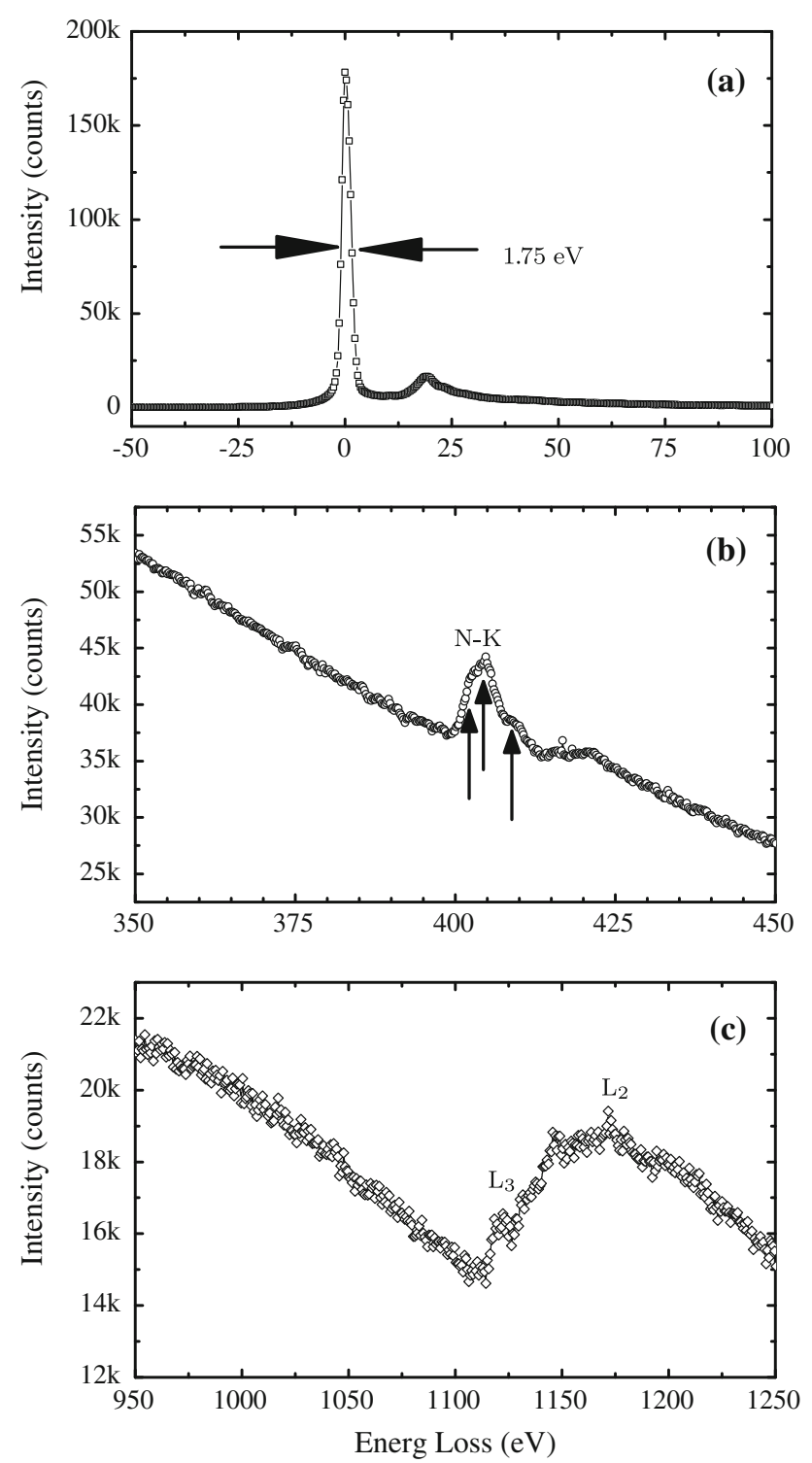

Fig. 3 EELS spectra of a GaN microtube. a Low loss EELS including the ZLP. ELNES of b nitrogen $\mathrm{K}$ edge and $\mathbf{c}$ gallium $\mathrm{L}_{2,3}$ edge. The arrows mark the main three peaks of $\mathrm{N}-\mathrm{K}$ edge

$550-750 \mathrm{~nm}$. The yellow band was usually observed in GaN nanocomposites [19, 20], nanoparticles [3], and nanowires [6]. It is generally accepted [27] that nitrogen vacancies and deep level impurities contribute to the yellow band. No any blue band was detected below $400 \mathrm{~nm}$ $(>3.1 \mathrm{eV})$.

The formation of the porous square microtubes may result from supersaturation growth, similar to that of tubular microtubes consisting of wurtize $\mathrm{GaN}$ nanoparticles [21]. It is known that gallium has a low melting point of $303 \mathrm{~K}$ and a boiling point of $2520 \mathrm{~K}$. Therefore large amounts of gallium droplets can form on the quartz substrates, as observed on silicon substrates [28]. The gallium droplets react with the flowing ammonia to produce $\mathrm{GaN}$

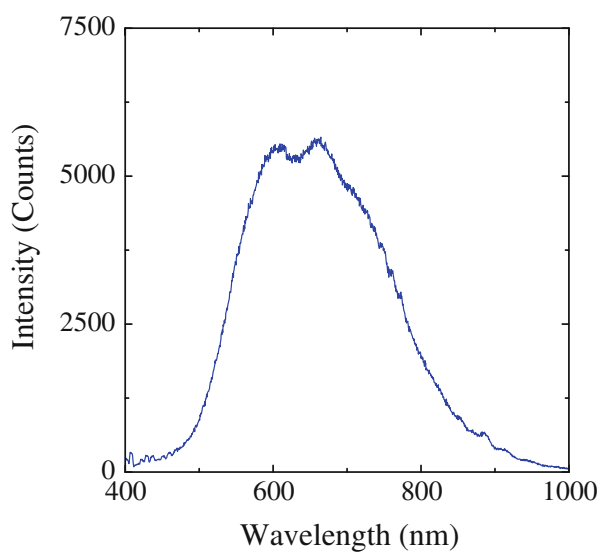

Fig. 4 PL spectrum of square microtubes

submicron irregular structures with a zincblende (cubic) structure. These zincblende $\mathrm{GaN}$ submicron irregular structures are so dense that they can be considered as a supersaturated aerosol, as observed in charged nanoparticles in solutions [29] or in vapor phases [30, 31]. The zincblende GaN submicron irregular structures then spontaneously aggregate into square microtubes.

It was reported that $\mathrm{h}-\mathrm{GaN}$ nanostructures with a hexagonal wurtzite structure self-assembled into circular microtubes [21], not square microtubes as observed here. At present we do know why c-GaN nanostructures selfassembled into square microtubes, while h-GaN nanostructures into circular microtubes. A most possible explanation is that $\mathrm{c}-\mathrm{GaN}$ nanostructures trend to square microtubes, while h-GaN nanostructures to circular microtubes in order to lower the total surface free energy of self-assembled micotubes. Thermodynamic calculations of the nanoporous microtubes consisting of $\mathrm{GaN}$ nanostructures should give more detailed information on total surface free energy of the microtubes and will be carried out in the future.

\section{Summary}

In a summary, a new kind of GaN self-assemblies was synthesized. The self-assemblies were nanoporous and square. These square microtubes were consisted of zincblende GaN submicron irregular structures and emitted broad yellow light centered at $550-750 \mathrm{~nm}$. A shift of $\mathrm{Ga}-\mathrm{L}_{2}$ electron-energy loss edge was observed in the GaN square microtubes.

Acknowledgements The authors thank the financial support by Defense Threat Reduction Agency under Grants HDTRA1-10-1-0001 and HDTRA122221, and the Center for Advanced Microscopy at Michigan State University for access to the JEM 2200 FS electron microscope. JMB acknowledges the support from National Science 
Foundation (CAREER Award ECCS-1240510 monitored by Anupama Kaul, DMR-0907336 monitored by Charles Ying), and from the Robert A Welch Foundation (E-1728). FL was supported by the National Basic Research Program of China (973 program), Grant No. 2012CB933004.

\section{References}

1. Ponce FA, Bour DP (1997) Nature 386:351. doi:10.1038/ $386351 \mathrm{a} 0$

2. Nakamura S (1998) Science 281(5379):956. doi:10.1126/science. 281.5379.956

3. Lan YC, Chen XL, Xu YP, Cao YG, Huang F (2000) Mater Res Bull 35(14-15):2325. doi:10.1016/S0025-5408(00)00447-5

4. Li S, Waag A (2012) J Appl Phys 111(7):071101/1. doi:10.1063/ 1.3694674

5. Li JY, Chen XL, Qiao ZY, Cao YG, Lan YC (2000) J Cryst Growth 213(3-4):408. doi:10.1016/S0022-0248(00)00390-0

6. Chen X, Li J, Cao Y, Lan Y, Li H, He M, Wang C, Zhang Z, Qiao Z (2000) Adv Mater 12(19):1432. doi:10.1002/15214095(200010)12:19<1432::AID-ADMA1432>3.0.CO;2-X

7. Kuykendall T, Pauzauskie P, Lee S, Zhang Y, Goldberger J, Yang P (2003) Nano Lett 3(8):1063. doi:10.1021/nl034422t

8. Bae SY, Seo HW, Park J, Yang H, Kim B (2003) Chem Phys Lett 376(3-4):445. doi:10.1016/S0009-2614(03)00955-2

9. Zimmler MA, Bao J, Shalish I, Yi W, Yoon J, Narayanamurti V, Capasso F (2007) Nanotechnology 18(23):235205. doi:10.1088/ 0957-4484/18/23/235205

10. Goldberger J, He R, Zhang Y, Lee S, Yan H, Choi HJ, Yang P (2003) Nature 422(6932):599. doi:10.1038/nature01551

11. He M, Minus I, Zhou P, Mohammed SN, Halpern JB, Jacobs R, Sarney WL, Salamanca-Riba L, Vispute RD (2000) Appl Phys Lett 77(23):3731. doi:10.1063/1.1329863

12. Hu JQ, Bando Y, Zhan JH, Xu FF, Sekiguchi T, Golberg D (2004) Adv Mater 16(16):1465. doi:10.1002/adma.200400016

13. Liu B, Bando Y, Tang C, Shen G, Golberg D, Xu F (2006) Appl Phys Lett 88(9):093120. doi:10.1063/1.2182065

14. Song Y, Wang W, Yuan W, Wu X, Chen X (2003) J Cryst Growth 247(34):275. doi:10.1016/S0022-0248(02)02014-6
15. Hashimoto T, Wu F, Speck JS, Nakamura S (2007) Nat Mater 6(8):568. doi:10.1038/nmat1955

16. Grzegory I, Boćkowski M, Lucznik B, Weyher J, Litwin-Staszewska E, Konczewicz L, Sadovyi B, Nowakowski P, Porowski S (2012) J Cryst Growth 350(1):50. doi:10.1016/j.jcrysgro.2011. 12.021

17. Wang D, Pierre A, Kibria MG, Cui K, Han X, Bevan KH, Guo H, Paradis S, Hakima AR, Mi Z (2011) Nano Lett 11(6):2353. doi:10.1021/nl2006802

18. Ryu SW, Zhang Y, Leung B, Yerino C, Han J (2012) Semicond Sci Technol 27(1):015014. doi:10.1088/0268-1242/27/1/015014

19. Chen XL, Cao YG, Lan YC, Xu XP, Li JQ, Lu KQ, Jiang PZ, Xu T, Bai ZG, Yu YD, Liang JK (2000) J Cryst Growth 209(1):208. doi:10.1016/S0022-0248(99)00522-9

20. Yin LW, Bando Y, Li MS, Golberg D (2005) Small 1(11):1094. doi:10.1002/smll.200500168

21. Lan Y, Lin F, Wang H, Ren Z, Crimp MA (2013) Adv Mater (submitted)

22. Lazar S, Botton G, Wu MY, Tichelaar F, Zandbergen H (2003) Ultramicroscopy 96(34):535. doi:10.1016/S0304-3991(03)00114-1

23. Bosman M, Tang LJ, Ye JD, Tan ST, Zhang Y, Keast VJ (2009) Appl Phys Lett 95(10):101110. doi:10.1063/1.3222974

24. Palisaitis J, Hsiao CL, Junaid M, Birch J, Hultman L, Persson POA (2011) Phys Rev B 84:245301. doi:10.1103/PhysRevB.84. 245301

25. Moreno M, Jorissen K, Rehr J. (2007) Micron 38(1):1. doi:10. 1016/j.micron.2006.03.011

26. Moreno MS, Lazar S, Zandbergen HW, Egerton RF (2006) Phys Rev B 73:073308. doi:10.1103/PhysRevB.73.073308

27. Reshchikov MA, Morkoç H (2005) J Appl Phys 97(6):061301. doi:10.1063/1.1868059

28. Sacilotti M, Imhoff L, Bourgeois S, Dumas C, Decobert J, Baldeck P, Colombier I (2004) J Cryst Growth 261(2-3):253. doi:10. 1016/j.jcrysgro.2003.11.065

29. Lagzi I, Kowalczyk B, Grzybowski BA (2010) J Am Chem Soc 132(1):58. doi:10.1021/ja906890v

30. Spotz EL, Hirschfelder JO (1951) J Chem Phys 19(9):1215. doi:10.1063/1.1748519

31. Goodeve CF, Eastman AS, Dooley A (1934) Trans Faraday Soc 30:1127. doi:10.1039/TF9343001127 